\title{
Single Oral Dose Toxicity Test of Low Molecular Weight Fucoidan in Rats
}

\author{
Hyun-Soo Yoon ${ }^{1}$, Yong-Kyu Shin ${ }^{2}$, Young-Mi Jung ${ }^{3}$, Hyeung-Sik LeE ${ }^{4}$, and Sae-Kwang Ku ${ }^{5, *}$ \\ ${ }^{1}$ Department of Anatomy and Cell Biology, Hanyang University College of Medicine, Seoul 133-791, ${ }^{2}$ BION Co., Ltd., Daegu 702-832, \\ ${ }^{3}$ Department of Genetic Engineering, College of Natural Sciences, Kyungpook National University, Daegu 702-701, \\ ${ }^{4}$ Department of Clinical Laboratory Science, College of Health and Therapy, ${ }^{5}$ Department of Anatomy and Histology, \\ College of Oriental Medicine, Daegu Haany University, Gyeongsan 712-715, Republic of Korea
}

(Received May 19, 2009; Revised June 12, 2009; Accepted July 4, 2009)

\begin{abstract}
The object of this study was to evaluate the single oral dose toxicity of Low Molecular Weight Fucoidan (LMF) in male and female rats. LMF was administered to female and male SD rats as an oral dose of 2,000, 1,000 and $500 \mathrm{mg} / \mathrm{kg}$ (body wt.). Animals were monitored for the mortality and changes in body weight, clinical signs and gross observation organ weight and histopathology of 14 principle organs were examined upon necropsy. As the results, no LMF treatment related mortalities, clinical signs, changes on the body and organ weights, gross and histopathological observations against 14 principle organs were detected up to $2,000 \mathrm{mg} / \mathrm{kg}$ in both female and male rats except for some sporadic findings not LMF treatment related toxicological signs. Therefore, $\mathrm{LD}_{50}(50 \%$ lethal dose) and approximate LD of LMF after single oral treatment in female and male rats were considered over $2,000 \mathrm{mg} / \mathrm{kg}$ - the limited dosages recommended by KFDA Guidelines [2005-60, 2005], respectively.
\end{abstract}

Keywords: Low molecular weight fucoidan, Single oral dose toxicity, Rat, Histopathology

\section{INTRODUCTION}

Fucoidans, the sulfated polysaccharides extracted from brown algae, were first isolated almost one century ago, contain substantial percentages of L-fucose and sulfate ester groups (Berteau and Mulloy, 2003). Fucoidan is of particular pharmacological interest because its non-animal origin, exhibits anti-inflammatory activities and potent modulation of connective tissue proteolysis (Senni et al., 2006). Ina addition, their anticoagulant and antithrombotic, antitumor, antiviral, anti-complement and anti-inflammatory activities (Be'ress et al., 1993; Patankar et al., 1993; Blondin et al., 1996; Haroun-Bouhedja et al., 2000; Bojakowski et al., 2001; Marais and Joseleau, 2001) have been reported. However, it is difficult to use because of its higher molecular weights; the absorption and bioavailability of high molecular weight fucoidan was relatively lower (Shimizu et al., 2005). Therefore, it has been researched to reduce the molecular weights (Colliec et al., 1991).

Since the pharmacological effects of fucoidan were varied with molecular weights, fucoidan generally divided low

${ }^{*}$ Corresponding author

Tel: +82-53-819-1549 Fax: +82-53-819-1269

E-mail: gucci200@hanmail.net
$(<10 \mathrm{kDa})$, middle $(10-10,000 \mathrm{kDa})$ and $(>10,000 \mathrm{kDa})$ high molecular fucoidan (Matsubara et al., 2005). Low molecular fucoidan (LMF) also showed various pharmacological effects like high molecular weight fucoidan (HMF) (Zemani et al., 2005; Alkhatib et al., 2006; Lake et al., 2006; Fréguin-Bouilland et al., 2007).

Although preclinical studies using HMF have been performed (Li et al., 2005); upto now, no detailed toxicological assessment of LMF has been reported except for mouse single dose toxicity test (Jung et al., 2008). The objective of this study was to obtain the primary safety information about LMF, obtained by acid hydrolysis of HMF from brown seaweed according to a previously published protocol (Nardella et al., 2000; Jung et al., 2007) on rats, to further clarify their safety for clinical use.

\section{MATERIALS AND METHODS}

\section{Animals and husbandry}

Each of twenty female and male SD rats (6 week old upon receipt, SLC, Japan) was used after acclimatization for 7 days. Animals were allocated five per polycarbonate cage in a temperature $\left(20-25^{\circ} \mathrm{C}\right)$ and humidity $(45-50 \%)$ controlled room. Light:dark cycle was $12 \mathrm{h:12}$ h, and feed 
(Samyang, Korea) and water were supplied free to access. All animals were overnight fasted before dosing and terminal necropsy. Animals were marked by picric acid.

\section{Preparation and administration of LMF}

The LMF, gift from BION Co. Ltd (Korea), was obtained by acid hydrolysis of HMF extracts from brown seaweed according to a protocol previously patented (Nardella et al., 2000; Jung et al., 2007). Based of previously reported analytical methods (Dubois et al., 1956; Farndale et al., 1986), the characteristics of LMF were: weight-average molecular mass, $5 \pm 0.6 \mathrm{kDa}$ (polydispersity 2.1); fucose content $43.1 \%(\mathrm{w} / \mathrm{w})$; galactose content $12.9 \%(\mathrm{w} / \mathrm{w})$, uronic acid content $2.4 \%(\mathrm{w} / \mathrm{w})$, sulfate content $28 \%(\mathrm{w} / \mathrm{w})$, protein content $5.4 \%(\mathrm{w} / \mathrm{w})$, moisture content $3.2 \%(\mathrm{w} / \mathrm{w})$, and ash content $5 \%(\mathrm{w} / \mathrm{w})$. FT-IR analysis of LMF revealed a close identity with HMF. Prepared LMF is light brownish-white powder, and stored in a desiccator to protect from light and humidity. LMF is well dissolved (clear brown solution) at least $200 \mathrm{mg} / \mathrm{ml}$ concentrations in distilled water. The test article was single orally administered at a dosage volume of $10 \mathrm{ml} / \mathrm{kg}$ using distilled water as vehicle at 2,000,1,000 and $500 \mathrm{mg} / \mathrm{kg}$ dose levels recommended by Korea Food and Drug Administration (2005).

\section{Abnormal behavior, clinical sign and body weight}

All abnormal clinical signs and behaviors were recorded before and after dosing at least twice a day based on the functional observational battery test (Irwin, 1968; Dourish, 1987). Body weights were measured on the day of dosing (Day 0) prior to treatment, 1, 2, 7, 13 and 14 days after dosing. In addition, to reduce the individual body weight differences of animals at treatment, body weight gains during Day 0-7, Day 7-13 and Day 0-13 was also calculated based on measured body weight at each point.

\section{Necropsy}

All animals were subjected to terminal necropsy at the end of experiment at day 14 after overnight fasting (about $18 \mathrm{hr}$, water was not restricted). Animals were euthanized by carbon dioxide and gross necropsy was performed.

\section{Organ weight measurements and sampling}

The absolute organ weight was measured and then relative organ weight ( $\%$ for body weight) was calculated. The following organs were collected for histopathological observation.

Measured and sampled organs: lung, heart, thymus, left kidney, left adrenal gland, spleen, left testis, left ovary, liver, splenic lobe of pancreas, brain, left epididymis, total uterus and left popliteal lymph node.

\section{Histopathology}

Samples were fixed in $10 \%$ neutral buffered formalin. After $18 \mathrm{~h}$ of fixation, paraffin embedding was conducted and $4 \mu \mathrm{m}$ sections were prepared by routine histological methods. Representative sections of each specified organs were stained with hematoxylin-eosin for light microscopical examination.

\section{Statistical analyses}

Multiple comparison tests for different dose groups were conducted. Variance homogeneity was examined using the Levene test. If the Levene test indicated no significant deviations from variance homogeneity, the obtain data were analyzed by one way ANOVA test followed by Tukey HSD test to determine which pairs of group comparison were significantly different. In case of significant deviations from variance homogeneity were observed at Levene test, a non-parametric comparison test, the Mann-Whitney U-Wilcoxon Rank Sum W test was conducted to determine the specific pairs of group comparison, which are significantly different. $L D_{50}$ and $95 \%$ confidence limits were calculated by Probit method. Statistical analyses were conducted using SPSS for Windows (Release 12.0K, SPSS Inc., USA) and a p-value of less than 0.05 was considered to be a significant difference. In addition, degree of clinical signs, gross and histopathological findings were subdivided into 3 degrees: $3+$ severe, $2+$ moderate, $1+$ slight.

\section{RESULTS}

\section{Mortality rate}

No sudden mortality was detected in all dose levels

Table I. Body weight gains after oral treatment of LMF

\begin{tabular}{llll}
\hline \multirow{2}{*}{ Group } & \multicolumn{3}{c}{ Intervals } \\
\cline { 2 - 4 } & \multicolumn{1}{c}{ Day 0 -7} & \multicolumn{1}{c}{ Day $7-13$} & Day 0-14 \\
\hline Male & & & \\
Vehicle control & $74.60 \pm 4.04$ & $40.80 \pm 9.09$ & $115.40 \pm 12.76$ \\
$2,000 \mathrm{mg} / \mathrm{kg}$ & $80.00 \pm 10.61$ & $40.20 \pm 10.26$ & $120.20 \pm 18.58$ \\
$1,000 \mathrm{mg} / \mathrm{kg}$ & $73.60 \pm 7.40$ & $37.20 \pm 10.13$ & $110.80 \pm 14.91$ \\
$500 \mathrm{mg} / \mathrm{kg}$ & $78.80 \pm 9.65$ & $35.80 \pm 8.58$ & $114.60 \pm 16.06$ \\
Female & & & \\
Vehicle control & $41.60 \pm 1.34$ & $16.60 \pm 2.07$ & $58.20 \pm 3.11$ \\
$2,000 \mathrm{mg} / \mathrm{kg}$ & $43.20 \pm 4.76$ & $15.40 \pm 3.58$ & $58.60 \pm 2.30$ \\
$1,000 \mathrm{mg} / \mathrm{kg}$ & $41.20 \pm 4.21$ & $14.40 \pm 4.83$ & $55.60 \pm 4.83$ \\
$500 \mathrm{mg} / \mathrm{kg}$ & $39.20 \pm 6.42$ & $15.80 \pm 5.93$ & $55.00 \pm 10.27$ \\
\hline
\end{tabular}

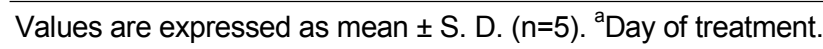




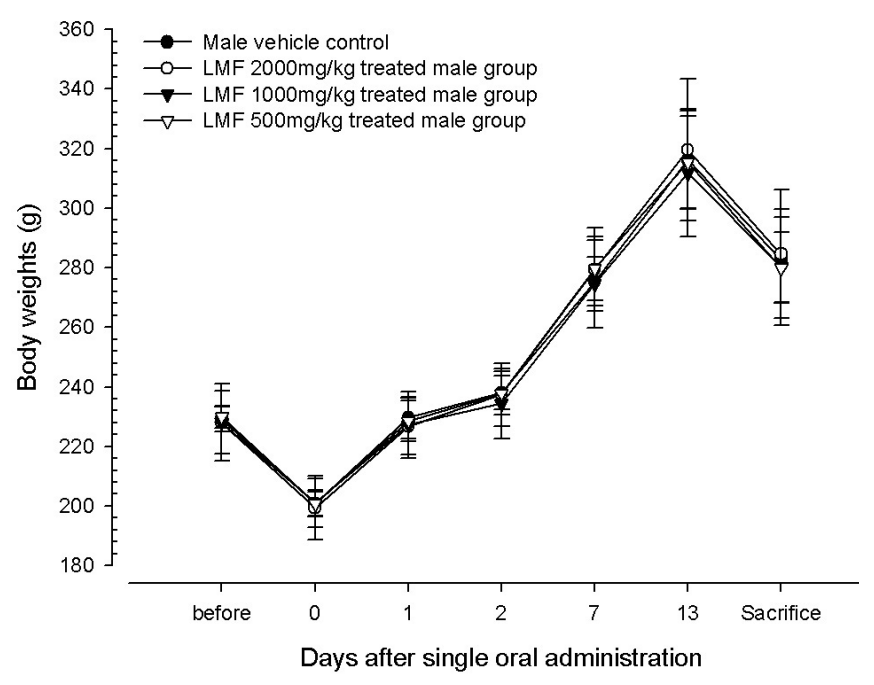

Fig. 1. Body weight changes in male rats after once oral administration of LMF. No meaningful changes were detected in all LMF treated groups as compared with vehicle control. Before means 1 day before administration, Day 0 means at administration, All animals at sacrifice and Day 0 overnight fasted.

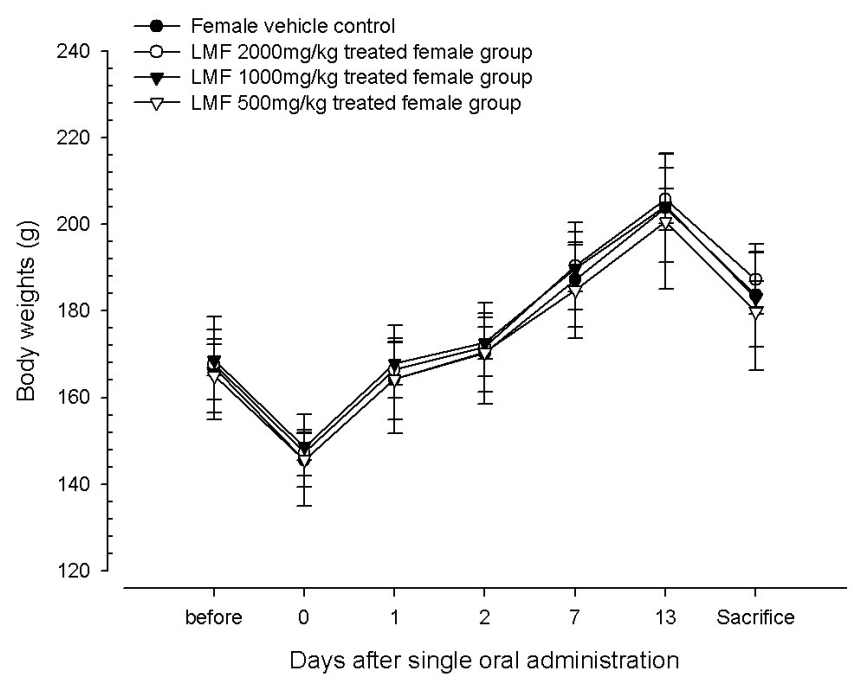

Fig. 2. Body weight changes in female rats after once oral administration of LMF. No meaningful changes were detected in all LMF treated groups as compared with vehicle control. Before means 1 day before administration, Day 0 means at administration, All animals at sacrifice and Day 0 overnight fasted.

Table II. Changes on the absolute organ weights after oral treatment of LMF

\begin{tabular}{|c|c|c|c|c|c|c|c|c|c|c|c|c|}
\hline \multirow[b]{2}{*}{ Group } & \multicolumn{12}{|c|}{ Organs: Male } \\
\hline & Lung & Heart & Thymus & Kidney L & $\begin{array}{l}\text { Adrenal } \\
\text { gland L }\end{array}$ & Spleen & Testis L & Liver & Pancreas S & Brain & $\begin{array}{l}\text { Epididy- } \\
\text { mis L }\end{array}$ & $\begin{array}{l}\text { Lymph } \\
\text { node } L^{a}\end{array}$ \\
\hline $\begin{array}{l}\text { Vehicle } \\
\text { control }\end{array}$ & $\begin{array}{c}1.236 \pm \\
0.060\end{array}$ & $\begin{array}{c}0.916 \pm \\
0.045\end{array}$ & $\begin{array}{c}0.478 \pm \\
0.091\end{array}$ & $\begin{array}{c}0.978 \pm \\
0.045\end{array}$ & $\begin{array}{c}0.044 \pm \\
0.011\end{array}$ & $\begin{array}{c}0.612 \pm \\
0.097\end{array}$ & $\begin{array}{c}1.742 \pm \\
0.082\end{array}$ & $\begin{array}{c}9.112 \pm \\
0.698\end{array}$ & $\begin{array}{c}0.584 \pm \\
0.065\end{array}$ & $\begin{array}{c}1.932 \pm \\
0.066\end{array}$ & $\begin{array}{c}0.394 \pm \\
0.044\end{array}$ & $\begin{array}{c}0.016 \pm \\
0.004\end{array}$ \\
\hline $\begin{array}{l}2,000 \\
\mathrm{mg} / \mathrm{kg}\end{array}$ & $\begin{array}{c}1.278 \pm \\
0.105\end{array}$ & $\begin{array}{c}0.934 \pm \\
0.085\end{array}$ & $\begin{array}{c}0.516 \pm \\
0.106\end{array}$ & $\begin{array}{c}0.985 \pm \\
0.100\end{array}$ & $\begin{array}{c}0.043 \pm \\
0.008\end{array}$ & $\begin{array}{c}0.615 \pm \\
0.090\end{array}$ & $\begin{array}{c}1.622 \pm \\
0.098\end{array}$ & $\begin{array}{c}8.987 \pm \\
0.763\end{array}$ & $\begin{array}{c}0.629 \pm \\
0.071\end{array}$ & $\begin{array}{c}1.904 \pm \\
0.065\end{array}$ & $\begin{array}{c}0.369 \pm \\
0.040\end{array}$ & $\begin{array}{c}0.013 \pm \\
0.006\end{array}$ \\
\hline $\begin{array}{l}1,000 \\
\mathrm{mg} / \mathrm{kg}\end{array}$ & $\begin{array}{c}1.223 \pm \\
0.106\end{array}$ & $\begin{array}{c}0.921 \pm \\
0.105\end{array}$ & $\begin{array}{c}0.495 \pm \\
0.103\end{array}$ & $\begin{array}{c}0.970 \pm \\
0.123\end{array}$ & $\begin{array}{c}0.048 \pm \\
0.015\end{array}$ & $\begin{array}{c}0.626 \pm \\
0.195\end{array}$ & $\begin{array}{c}1.716 \pm \\
0.204\end{array}$ & $\begin{array}{c}8.678 \pm \\
1.060\end{array}$ & $\begin{array}{c}0.673 \pm \\
0.087\end{array}$ & $\begin{array}{c}1.905 \pm \\
0.039\end{array}$ & $\begin{array}{c}0.417 \pm \\
0.044\end{array}$ & $\begin{array}{c}0.013 \pm \\
0.004\end{array}$ \\
\hline \multirow[t]{3}{*}{$\begin{array}{l}500 \\
\mathrm{mg} / \mathrm{kg}\end{array}$} & $\begin{array}{c}1.289 \pm \\
0.126\end{array}$ & $\begin{array}{c}0.983 \pm \\
0.140\end{array}$ & $\begin{array}{c}0.525 \pm \\
0.110\end{array}$ & $\begin{array}{c}1.012 \pm \\
0.077\end{array}$ & $\begin{array}{c}0.044 \pm \\
0.006\end{array}$ & $\begin{array}{c}0.553 \pm \\
0.113\end{array}$ & $\begin{array}{c}1.730 \pm \\
0.088\end{array}$ & $\begin{array}{c}8.958 \pm \\
0.730\end{array}$ & $\begin{array}{c}0.634 \pm \\
0.085\end{array}$ & $\begin{array}{c}1.913 \pm \\
0.094\end{array}$ & $\begin{array}{c}0.399 \pm \\
0.063\end{array}$ & $\begin{array}{c}0.019 \pm \\
0.006\end{array}$ \\
\hline & \multicolumn{12}{|c|}{ Organs: Female } \\
\hline & Lung & Heart & Thymus & Kidney L & $\begin{array}{l}\text { Adrenal } \\
\text { gland L }\end{array}$ & Spleen & Ovary L & Liver & Pancreas S & Brain & Uterus & $\begin{array}{l}\text { Lymph } \\
\text { node L }\end{array}$ \\
\hline $\begin{array}{l}\text { Vehicle } \\
\text { control }\end{array}$ & $\begin{array}{c}1.024 \pm \\
0.105\end{array}$ & $\begin{array}{c}0.669 \pm \\
0.053\end{array}$ & $\begin{array}{c}0.467 \pm \\
0.041\end{array}$ & $\begin{array}{c}0.686 \pm \\
0.076\end{array}$ & $\begin{array}{c}0.043 \pm \\
0.005\end{array}$ & $\begin{array}{c}0.458 \pm \\
0.072\end{array}$ & $\begin{array}{c}0.071 \pm \\
0.013\end{array}$ & $\begin{array}{c}5.905 \pm \\
0.525\end{array}$ & $\begin{array}{c}0.477 \pm \\
0.026\end{array}$ & $\begin{array}{c}1.863 \pm \\
0.109\end{array}$ & $\begin{array}{c}0.512 \pm \\
0.228\end{array}$ & $\begin{array}{c}0.016 \pm \\
0.004\end{array}$ \\
\hline $\begin{array}{l}2,000 \\
\mathrm{mg} / \mathrm{kg}\end{array}$ & $\begin{array}{c}0.997 \pm \\
0.045\end{array}$ & $\begin{array}{c}0.698 \pm \\
0.033\end{array}$ & $\begin{array}{c}0.436 \pm \\
0.017\end{array}$ & $\begin{array}{c}0.664 \pm \\
0.040\end{array}$ & $\begin{array}{c}0.049 \pm \\
0.007\end{array}$ & $\begin{array}{c}0.412 \pm \\
0.038\end{array}$ & $\begin{array}{c}0.064 \pm \\
0.011\end{array}$ & $\begin{array}{c}5.828 \pm \\
0.111\end{array}$ & $\begin{array}{c}0.484 \pm \\
0.044\end{array}$ & $\begin{array}{c}1.860 \pm \\
0.072\end{array}$ & $\begin{array}{c}0.403 \pm \\
0.175\end{array}$ & $\begin{array}{c}0.019 \pm \\
0.008\end{array}$ \\
\hline $\begin{array}{l}1,000 \\
\mathrm{mg} / \mathrm{kg}\end{array}$ & $\begin{array}{c}1.038 \pm \\
0.051\end{array}$ & $\begin{array}{c}0.691 \pm \\
0.050\end{array}$ & $\begin{array}{c}0.410 \pm \\
0.038\end{array}$ & $\begin{array}{c}0.667 \pm \\
0.032\end{array}$ & $\begin{array}{c}0.041 \pm \\
0.006\end{array}$ & $\begin{array}{c}0.416 \pm \\
0.043\end{array}$ & $\begin{array}{c}0.083 \pm \\
0.013\end{array}$ & $\begin{array}{c}5.640 \pm \\
0.246\end{array}$ & $\begin{array}{c}0.444 \pm \\
0.018\end{array}$ & $\begin{array}{c}1.853 \pm \\
0.046\end{array}$ & $\begin{array}{c}0.428 \pm \\
0.116\end{array}$ & $\begin{array}{c}0.014 \pm \\
0.003\end{array}$ \\
\hline $\begin{array}{l}500 \\
\mathrm{mg} / \mathrm{kg}\end{array}$ & $\begin{array}{c}0.986 \pm \\
0.072\end{array}$ & $\begin{array}{c}0.635 \pm \\
0.029\end{array}$ & $\begin{array}{c}0.439 \pm \\
0.070\end{array}$ & $\begin{array}{c}0.630 \pm \\
0.042\end{array}$ & $\begin{array}{c}0.050 \pm \\
0.007\end{array}$ & $\begin{array}{c}0.387 \pm \\
0.082\end{array}$ & $\begin{array}{c}0.070 \pm \\
0.011\end{array}$ & $\begin{array}{c}5.429 \pm \\
0.399\end{array}$ & $\begin{array}{c}0.463 \pm \\
0.046\end{array}$ & $\begin{array}{c}1.779 \pm \\
0.049\end{array}$ & $\begin{array}{c}0.366 \pm \\
0.041\end{array}$ & $\begin{array}{c}0.016 \pm \\
0.009\end{array}$ \\
\hline
\end{tabular}

Values are expressed as mean \pm S. D. $(n=5)$. L: left sides, S: splenic lobes. ${ }^{\mathrm{a}}$ Popliteal lymph node. 
tested. All animals $(5 / 5 ; 100 \%)$ survived in all dose levels tested including vehicle control.

\section{Abnormal clinical signs detected}

In this study, no LMF treatment related abnormal clinical signs were observed during observation periods regardless of male and female mice.

\section{Changes of body weights}

No significant changes in body weight were detected

Table III. Changes on the relative organ weights after oral treatment of LMF

\begin{tabular}{|c|c|c|c|c|c|c|c|c|c|c|c|c|}
\hline \multirow[b]{2}{*}{ Group } & \multicolumn{12}{|c|}{ Organs: Male } \\
\hline & Lung & Heart & Thymus & Kidney L & $\begin{array}{l}\text { Adrenal } \\
\text { gland L }\end{array}$ & Spleen & Testis L & Liver & Pancreas S & Brain & $\begin{array}{l}\text { Epididy- } \\
\text { mis L }\end{array}$ & $\begin{array}{l}\text { Lymph } \\
\text { node } L^{a}\end{array}$ \\
\hline $\begin{array}{l}\text { Vehicle } \\
\text { control }\end{array}$ & $\begin{array}{c}0.439 \pm \\
0.037\end{array}$ & $\begin{array}{c}0.324 \pm \\
0.015\end{array}$ & $\begin{array}{c}0.169 \pm \\
0.029\end{array}$ & $\begin{array}{c}0.346 \pm \\
0.016\end{array}$ & $\begin{array}{c}0.016 \pm \\
0.004\end{array}$ & $\begin{array}{c}0.216 \pm \\
0.028\end{array}$ & $\begin{array}{c}0.617 \pm \\
0.030\end{array}$ & $\begin{array}{c}3.221 \pm \\
0.103\end{array}$ & $\begin{array}{c}0.207 \pm \\
0.019\end{array}$ & $\begin{array}{c}0.685 \pm \\
0.033\end{array}$ & $\begin{array}{c}0.140 \pm \\
0.019\end{array}$ & $\begin{array}{c}0.006 \pm \\
0.001\end{array}$ \\
\hline $\begin{array}{l}2,000 \\
\mathrm{mg} / \mathrm{kg}\end{array}$ & $\begin{array}{c}0.449 \pm \\
0.010\end{array}$ & $\begin{array}{c}0.329 \pm \\
0.029\end{array}$ & $\begin{array}{c}0.180 \pm \\
0.027\end{array}$ & $\begin{array}{c}0.346 \pm \\
0.013\end{array}$ & $\begin{array}{c}0.015 \pm \\
0.003\end{array}$ & $\begin{array}{c}0.216 \pm \\
0.021\end{array}$ & $\begin{array}{c}0.571 \pm \\
0.033\end{array}$ & $\begin{array}{c}3.156 \pm \\
0.067\end{array}$ & $\begin{array}{c}0.223 \pm \\
0.036\end{array}$ & $\begin{array}{c}0.671 \pm \\
0.037\end{array}$ & $\begin{array}{c}0.130 \pm \\
0.012\end{array}$ & $\begin{array}{c}0.004 \pm \\
0.002\end{array}$ \\
\hline $\begin{array}{l}1,000 \\
\mathrm{mg} / \mathrm{kg}\end{array}$ & $\begin{array}{c}0.436 \pm \\
0.016\end{array}$ & $\begin{array}{c}0.329 \pm \\
0.028\end{array}$ & $\begin{array}{c}0.176 \pm \\
0.032\end{array}$ & $\begin{array}{c}0.345 \pm \\
0.021\end{array}$ & $\begin{array}{c}0.017 \pm \\
0.005\end{array}$ & $\begin{array}{c}0.221 \pm \\
0.056\end{array}$ & $\begin{array}{c}0.612 \pm \\
0.056\end{array}$ & $\begin{array}{c}3.088 \pm \\
0.173\end{array}$ & $\begin{array}{c}0.240 \pm \\
0.019\end{array}$ & $\begin{array}{c}0.682 \pm \\
0.051\end{array}$ & $\begin{array}{c}0.149 \pm \\
0.013\end{array}$ & $\begin{array}{c}0.005 \pm \\
0.001\end{array}$ \\
\hline \multirow[t]{3}{*}{$\begin{array}{l}5,00 \\
\mathrm{mg} / \mathrm{kg}\end{array}$} & $\begin{array}{c}0.460 \pm \\
0.032\end{array}$ & $\begin{array}{c}0.351 \pm \\
0.047\end{array}$ & $\begin{array}{c}0.187 \pm \\
0.032\end{array}$ & $\begin{array}{c}0.362 \pm \\
0.028\end{array}$ & $\begin{array}{c}0.016 \pm \\
0.003\end{array}$ & $\begin{array}{c}0.197 \pm \\
0.034\end{array}$ & $\begin{array}{c}0.619 \pm \\
0.042\end{array}$ & $\begin{array}{c}3.196 \pm \\
0.162\end{array}$ & $\begin{array}{c}0.226 \pm \\
0.022\end{array}$ & $\begin{array}{c}0.683 \pm \\
0.021\end{array}$ & $\begin{array}{c}0.143 \pm \\
0.026\end{array}$ & $\begin{array}{c}0.007 \pm \\
0.002\end{array}$ \\
\hline & \multicolumn{12}{|c|}{ Organs: Female } \\
\hline & Lung & Heart & Thymus & Kidney L & $\begin{array}{l}\text { Adrenal } \\
\text { gland L }\end{array}$ & Spleen & Ovary L & Liver & Pancreas S & Brain & Uterus & $\begin{array}{l}\text { Lymph } \\
\text { node L }\end{array}$ \\
\hline $\begin{array}{l}\text { Vehicle } \\
\text { control }\end{array}$ & $\begin{array}{c}0.557 \pm \\
0.031\end{array}$ & $\begin{array}{c}0.367 \pm \\
0.050\end{array}$ & $\begin{array}{c}0.255 \pm \\
0.030\end{array}$ & $\begin{array}{c}0.373 \pm \\
0.027\end{array}$ & $\begin{array}{c}0.023 \pm \\
0.002\end{array}$ & $\begin{array}{c}0.250 \pm \\
0.043\end{array}$ & $\begin{array}{c}0.039 \pm \\
0.008\end{array}$ & $\begin{array}{c}3.215 \pm \\
0.159\end{array}$ & $\begin{array}{c}0.261 \pm \\
0.027\end{array}$ & $\begin{array}{c}1.016 \pm \\
0.043\end{array}$ & $\begin{array}{c}0.276 \pm \\
0.112\end{array}$ & $\begin{array}{c}0.009 \pm \\
0.002\end{array}$ \\
\hline $\begin{array}{l}2,000 \\
\mathrm{mg} / \mathrm{kg}\end{array}$ & $\begin{array}{c}0.532 \pm \\
0.016\end{array}$ & $\begin{array}{c}0.373 \pm \\
0.017\end{array}$ & $\begin{array}{c}0.233 \pm \\
0.005\end{array}$ & $\begin{array}{c}0.355 \pm \\
0.013\end{array}$ & $\begin{array}{c}0.026 \pm \\
0.004\end{array}$ & $\begin{array}{c}0.220 \pm \\
0.014\end{array}$ & $\begin{array}{c}0.034 \pm \\
0.005\end{array}$ & $\begin{array}{c}3.115 \pm \\
0.052\end{array}$ & $\begin{array}{c}0.259 \pm \\
0.024\end{array}$ & $\begin{array}{c}0.996 \pm \\
0.071\end{array}$ & $\begin{array}{c}0.215 \pm \\
0.091\end{array}$ & $\begin{array}{c}0.010 \pm \\
0.004\end{array}$ \\
\hline $\begin{array}{l}1,000 \\
\mathrm{mg} / \mathrm{kg}\end{array}$ & $\begin{array}{c}0.568 \pm \\
0.031\end{array}$ & $\begin{array}{c}0.378 \pm \\
0.028\end{array}$ & $\begin{array}{c}0.224 \pm \\
0.021\end{array}$ & $\begin{array}{c}0.365 \pm \\
0.017\end{array}$ & $\begin{array}{c}0.023 \pm \\
0.004\end{array}$ & $\begin{array}{c}0.227 \pm \\
0.023\end{array}$ & $\begin{array}{c}0.046 \pm \\
0.006\end{array}$ & $\begin{array}{c}3.083 \pm \\
0.161\end{array}$ & $\begin{array}{c}0.243 \pm \\
0.005\end{array}$ & $\begin{array}{c}1.013 \pm \\
0.011\end{array}$ & $\begin{array}{c}0.234 \pm \\
0.065\end{array}$ & $\begin{array}{c}0.008 \pm \\
0.002\end{array}$ \\
\hline $\begin{array}{l}500 \\
\mathrm{mg} / \mathrm{kg}\end{array}$ & $\begin{array}{c}0.549 \pm \\
0.011\end{array}$ & $\begin{array}{c}0.354 \pm \\
0.016\end{array}$ & $\begin{array}{c}0.243 \pm \\
0.022\end{array}$ & $\begin{array}{c}0.351 \pm \\
0.005\end{array}$ & $\begin{array}{c}0.028 \pm \\
0.005\end{array}$ & $\begin{array}{c}0.214 \pm \\
0.037\end{array}$ & $\begin{array}{c}0.039 \pm \\
0.006\end{array}$ & $\begin{array}{c}3.020 \pm \\
0.019^{b}\end{array}$ & $\begin{array}{c}0.258 \pm \\
0.023\end{array}$ & $\begin{array}{c}0.993 \pm \\
0.065\end{array}$ & $\begin{array}{c}0.203 \pm \\
0.012\end{array}$ & $\begin{array}{c}0.009 \pm \\
0.005\end{array}$ \\
\hline
\end{tabular}

Values are expressed as mean \pm S. D., $\%$ of body weight at sacrifice $(n=5)$. L: left sides, S: splenic lobes, ${ }^{a}$ Popliteal lymph node, ${ }^{b} p$ $<0.05$ as compared with vehicle control.

Table IV. Necropsy findings after oral treatment of LMF

\begin{tabular}{|c|c|c|c|c|c|c|c|c|}
\hline \multirow[b]{2}{*}{ Group } & \multicolumn{4}{|c|}{ Male } & \multicolumn{4}{|c|}{ Female } \\
\hline & $\begin{array}{l}\text { Vehicle } \\
\text { control }\end{array}$ & $2,000 \mathrm{mg} / \mathrm{kg}$ & $1,000 \mathrm{mg} / \mathrm{kg}$ & $500 \mathrm{mg} / \mathrm{kg}$ & $\begin{array}{l}\text { Vehicle } \\
\text { control }\end{array}$ & $2,000 \mathrm{mg} / \mathrm{kg}$ & $1,000 \mathrm{mg} / \mathrm{kg}$ & $500 \mathrm{mg} / \mathrm{kg}$ \\
\hline \multicolumn{9}{|l|}{ Lung } \\
\hline Normal & $4 / 5$ & $4 / 5$ & $5 / 5$ & $5 / 5$ & $3 / 5$ & $4 / 5$ & $4 / 5$ & $4 / 5$ \\
\hline Congestion & $1 / 5$ & $1 / 5$ & $0 / 5$ & $0 / 5$ & $2 / 5$ & $1 / 5$ & $1 / 5$ & $1 / 5$ \\
\hline \multicolumn{9}{|l|}{ Thymus } \\
\hline Normal & $4 / 5$ & $3 / 5$ & $4 / 5$ & $5 / 5$ & $4 / 5$ & $5 / 5$ & $4 / 5$ & $3 / 5$ \\
\hline Atrophy & $1 / 5$ & $1 / 5$ & $1 / 5$ & $0 / 5$ & $0 / 5$ & $0 / 5$ & $0 / 5$ & $1 / 5$ \\
\hline Congestion & $0 / 5$ & $1 / 5$ & $0 / 5$ & $0 / 5$ & $1 / 5$ & $0 / 5$ & $1 / 5$ & $1 / 5$ \\
\hline \multicolumn{9}{|l|}{ Spleen } \\
\hline Normal & $4 / 5$ & $5 / 5$ & $3 / 5$ & $4 / 5$ & $4 / 5$ & $5 / 5$ & $4 / 5$ & $4 / 5$ \\
\hline Atrophy & $1 / 5$ & $0 / 5$ & $2 / 5$ & $1 / 5$ & $1 / 5$ & $0 / 5$ & $1 / 5$ & $1 / 5$ \\
\hline \multicolumn{9}{|l|}{ Epididymis/Uterus } \\
\hline Normal & $5 / 5$ & $5 / 5$ & $5 / 5$ & $5 / 5$ & $4 / 5$ & $5 / 5$ & $3 / 5$ & $4 / 5$ \\
\hline Edematous changes & $0 / 5$ & $0 / 5$ & $0 / 5$ & $0 / 5$ & $1 / 5$ & $0 / 5$ & $2 / 5$ & $1 / 5$ \\
\hline
\end{tabular}

Observed animals/total observed animals $(n=5)$. 
Table V. Histopathological findings after oral treatment of LMF

\begin{tabular}{|c|c|c|c|c|c|c|c|c|}
\hline \multirow[b]{2}{*}{ Group } & \multicolumn{4}{|c|}{ Male } & \multicolumn{4}{|c|}{ Female } \\
\hline & $\begin{array}{l}\text { Vehicle } \\
\text { control }\end{array}$ & $2,000 \mathrm{mg} / \mathrm{kg}$ & $1,000 \mathrm{mg} / \mathrm{kg}$ & $500 \mathrm{mg} / \mathrm{kg}$ & $\begin{array}{l}\text { Vehicle } \\
\text { control }\end{array}$ & $2,000 \mathrm{mg} / \mathrm{kg}$ & $1,000 \mathrm{mg} / \mathrm{kg}$ & $500 \mathrm{mg} / \mathrm{kg}$ \\
\hline \multicolumn{9}{|l|}{ Lung } \\
\hline Normal & $4 / 5$ & $3 / 5$ & $4 / 5$ & $4 / 5$ & $3 / 5$ & $4 / 5$ & $5 / 5$ & $4 / 5$ \\
\hline Congestion & $1 / 5$ & $2 / 5$ & $1 / 5$ & $1 / 5$ & $2 / 5$ & $1 / 5$ & $0 / 5$ & $1 / 5$ \\
\hline \multicolumn{9}{|l|}{ Thymus } \\
\hline Normal & $5 / 5$ & $4 / 5$ & $4 / 5$ & $5 / 5$ & $4 / 5$ & $5 / 5$ & $5 / 5$ & $3 / 5$ \\
\hline FC (Medulla) & $0 / 5$ & $1 / 5$ & $1 / 5$ & $0 / 5$ & $0 / 5$ & $0 / 5$ & $0 / 5$ & $2 / 5$ \\
\hline FH (Cortex) & $0 / 5$ & $0 / 5$ & $0 / 5$ & $0 / 5$ & $1 / 5$ & $0 / 5$ & $0 / 5$ & $0 / 5$ \\
\hline \multicolumn{9}{|l|}{ Adrenal gland } \\
\hline Normal & $4 / 5$ & $4 / 5$ & $5 / 5$ & $5 / 5$ & $5 / 5$ & $5 / 5$ & $5 / 5$ & $5 / 5$ \\
\hline FC (Cortex) & $1 / 5$ & $1 / 5$ & $0 / 5$ & $0 / 5$ & $0 / 5$ & $0 / 5$ & $0 / 5$ & $0 / 5$ \\
\hline \multicolumn{9}{|l|}{ Liver } \\
\hline Normal & $5 / 5$ & $5 / 5$ & $4 / 5$ & $4 / 5$ & $4 / 5$ & $5 / 5$ & $4 / 5$ & $5 / 5$ \\
\hline IF & $0 / 5$ & $0 / 5$ & $1 / 5$ & $1 / 5$ & $1 / 5$ & $0 / 5$ & $1 / 5$ & $0 / 5$ \\
\hline \multicolumn{9}{|c|}{ Popliteal lymph node } \\
\hline Normal & $5 / 5$ & $5 / 5$ & $4 / 5$ & $4 / 5$ & $4 / 5$ & $5 / 5$ & $5 / 5$ & $5 / 5$ \\
\hline $\mathrm{DE}$ & $0 / 5$ & $0 / 5$ & $1 / 5$ & $1 / 5$ & $1 / 5$ & $0 / 5$ & $0 / 5$ & $0 / 5$ \\
\hline
\end{tabular}

Observed animals/total observed animals $(n=5)$. IF: focal inflammatory cell infiltration, FC: focal congestion, FH: focal hemorrhages, DE: decreases of lymphoid cells in medullary sinus.

compared to that of vehicle control in all dose levels tested (Table I, Fig. 1, 2).

\section{Changes of organ weights}

No meaningful changes on the absolute and relative organ weight of 14 principle organs were observed in all LMF treated female and male rats as compared with each equal gender of vehicle control, except for significant $(p<0.05)$ decreases in liver relative weight restricted to the lowest dosage, $500 \mathrm{mg} / \mathrm{kg}$ of LMF treated female rats as compared with female vehicle control in the present study (Table II, III).

\section{Necropsy findings}

No LMF-treatment related changes on the gross findings were observed as compared with equal gender of vehicle control except for some sporadic findings such as slight $(1+)$ congestion of lung, thymic atrophy or congestion, splenic atrophy and edematous changes of uterus, which were sporadically detected throughout all experimental groups tested in the present including both gender of vehicle control (Table IV).

\section{Histopathological findings}

No meaningful changes on the histopathological findings of 14 principle organs were observed in LMF-treated groups as compared with equal gender of vehicle control except for some sporadic findings such as slight $(1+)$ lung congestional spots - thickening of alveolar wall with inflammatory cell infiltration and focal hemorrhages, focal medullar congestion or cortex hemorrhage of thymus, focal cortical congestion of adrenal glands, focal inflammatory cell infiltrations of liver parenchyma and decreases of lymphoid cells in the medullary sinus of popliteal lymph nodes, which were sporadically detected throughout all experimental groups tested in the present study including both gender vehicle controls (Table V).

\section{DISCUSSION}

In the present study, we investigated the single oral dose toxicity of LMF on the rats as a part of the safety test. In order to observe $50 \%$ lethal dose $\left(L_{50}\right)$ and approximate lethal dosage (LD), test substances were administered orally to female and male SD rats at dose levels of $2,000,1,000$ and $500 \mathrm{mg} / \mathrm{kg}$. We could not find any mortality, clinical signs, and changes in the body and organ weight except for significant $(p<0.05)$ decreases of liver relative weights restricted to the $500 \mathrm{mg} / \mathrm{kg}$ of LMF treated female group as compared with female vehicle control. In addition, no LMF-treatment related abnormal gross findings and changes in histopathology of principle organs were detected except for some sporadic accidental findings in both male and female rats.

No LMF treatment related mortalities and clinical signs were detected up to $2,000 \mathrm{mg} / \mathrm{kg}$ - the highest dosages 
used in the present study. In addition, all animals including $2,000 \mathrm{mg} / \mathrm{kg}$ treated male and female rats shows body weight increases ranged in normal age-matched rats (Fox et al., 1984; Tajima, 1989) regardless of treatment in the present study. Decreases of relative liver weights restricted to the lowest female dosage group as compared with female vehicle control, is not considered as LMF treatment related toxicological signs because they did not showed any dosage-dependent changes and no meaningful gross and histopathological changes were detected in the liver in the present study. The slight congestion of lung, thymic atrophy and congestion, splenic atrophy and edematous changes of the uterus detected as gross findings, and congestional spots in lung, focal thymic congestion or hemorrhages, adrenal gland congestion, focal inflammatory cell infiltrations of liver parenchyma and decreases of lymphoid cells in the medullary sinus of popliteal lymph nodes detected as histopathological findings were considered as accidental findings not toxicological signs related to the LMF treatment because they were sporadically detected throughout the whole experimental groups tested in the present study including both genders of vehicle control. Especially, the edematous changes in uterus were considered as secondary changes from different physiological estrus cycles (Banks, 1986; Pineda, 1989). In addition, most of them were also generally observed in normal rats (Boorman et al., 1990; Greaves, 1990; Hasechek and Rousseaux, 1998).

Well corresponded to the results of previous 4 weeks repeated oral toxicity test of HMF (Li et al., 2005) and mouse single oral dose toxicity test of LMF (Jung et al., 2008), no LMF treatment related mortalities were detected up to $2,000 \mathrm{mg} / \mathrm{kg}$ in both male and female rats, the $\mathrm{LD}_{50}$ and approximate LD of LMF after single oral treatment in female and male rats were considered over $2,000 \mathrm{mg} / \mathrm{kg}$, respectively and is likely to be safe in humans.

\section{REFERENCES}

Alkhatib, B., Freguin-Bouilland, C., Lallemand, F., Henry, J. P., Litzler, P. Y., Marie, J. P., Richard, V., Thuillez, C. and Plissonnier, D. (2006). Low molecular weight fucan prevents transplant coronaropathy in rat cardiac allograft model. Transpl. Immunol. 16, 14-19.

Banks, W. J. (1986). Female reproductive system. In Applied veterinary histology (W. J. Banks, Ed.), pp. 506-526. Williams \& Wilkins, Baltimore.

Be'ress, A., Wassermann, O., Tahhan, S., Bruhn, T., Beress, L. and Kraiselburd, E. N. (1993). A new procedure for the isolation of anti-HIV compounds (polysaccharide and polyphenols) from the marine algae Fucus vesiculosus. J. Nat. Prod. 56, 478-488.
Berteau, O. and Mulloy, B. (2003). Sulfated fucans, fresh perspectives: structures, functions, and biological properties of sulfated fucans and an overview of enzymes active toward this class of polysaccharide. Glycobiology 13, 29R-40R.

Blondin, C., Chaubet, F., Nardella, A., Sinquin, C. and Jozefonvicz, J. (1996). Relationships between chemical characteristics and anticomplementary activity of fucans. Biomaterials 17, 597-603.

Bojakowski, K., Abramczyk, P., Bojakowska, M., Zwolinska, A., Przybylski, J. and Gaciong, Z. (2001). Fucoidan improves the renal blood flow in the early stage of renal ischemia/reperfusion injury in the rat. J. Physiol. Pharmacol. 52, 137-143.

Boorman, G. A., Eustis, S. L., Elwell, M. R., Montgomery, C. A. Jr. and Mackenzie, W. F. (1990). Pathology of the Fischer Rat. Reference and Atlas. Academic Press, San Diego.

Colliec, S., Fischer, A. M., Tapon-Bretaudiere, J., Boisson, C., Durand, P. and Jozefonvicz, J. (1991). Anticoagulant properties of a fucoidan fraction. Thromb. Res. 64, 143-154.

Dourish, C. T. (1987). Effects of drugs on spontaneous motor activity. In Experimental Psychopharmacology (A. J. Greenshaw and C. T. Dourish, Ed.), pp. 325-334. Humana Press, Clifton.

Dubois, M., Gilles, K. A., Hamilton, J. K., Rebers, P. A. and Smith, F. (1956). Colorimetric method for determination of sugars and related substances. Anal. Chem. 28, 350-354.

Farndale, R. W., Buttle, D. J. and Barret, A. J. (1986). Improved quantification and discrimination of sulphated glycosaminoglycans by use of dimethylene blue. Biochim. Biophys. Acta. 883, 173-177.

Fox, J. G., Cohen, B. J. and Loew, F. M. (1984). Laboratory animal medicine. Academic Press, Orlando.

Fréguin-Bouilland, C., Alkhatib, B., David, N., Lallemand, F., Henry, J. P., Godin, M., Thuillez, C. and Plissonnier, D. (2007). Low molecular weight fucoidan prevents neointimal hyperplasia after aortic allografting. Transplantation $\mathbf{8 3}$, 1234-1241.

Greaves, P. (1990). Histopathology of Preclinical Studies: Interpretation and Relevance in Drug Evaluation. Elsevier, New York.

Haroun-Bouhedja, F., Ellouali, M., Sinquin, C. and BoissonVidal, C. (2000). Relationship between sulfate groups and biological activities of fucans. Thromb. Res. 100, 453-459.

Hasechek, W. M. and Rousseaux, C. G. (1998). Fundamanentals of Toxicologic Pathology. Academic Press, San Diego.

Irwin, S. (1968). Comprehensive observational assessment: la. A systemic, quantitative procedure for assessing the behavioral and physiological state of the mouse. Psychopharmacology 13, 222-257.

Jung, Y. M., Kim, T. K., Park, D. C. and Lee, Y. H. (2007). Bioavailable Fucoidan and Methods for Preparing the Same, Korean Patent 0705975.

Jung, Y. M., Yoo, K. M., Park, D. C., Kim, T. K., Lee, H. S. and Ku S. K. (2008). Oral single dose toxicity study of low molecular fucoidan in mice. Toxicol. Res. 24, 79-86.

Korea Food and Drug Administration. (2005). Testing Guidelines for Safety Evaluation of Drugs (Notification No. 200560 , issued by the Korea Food and Drug Administration on October 21, 2005).

Lake, A. C., Vassy, R., Di Benedetto, M., Lavigne, D., Le 
Visage, C., Perret, G. Y. and Letourneur, D. (2006). Low molecular weight fucoidan increases VEGF165-induced endothelial cell migration by enhancing VEGF165 binding to VEGFR-2 and NRP1. J. Biol. Chem. 281, 37844-37852.

Li, N., Zhang, Q. and Song, J. (2005). Toxicological evaluation of fucoidan extracted from Laminaria japonica in Wistar rats. Food Chem. Toxicol. 43, 421-426.

Marais, M. F. and Joseleau, J. P. (2001). A fucoidan fraction from Ascophyllum nodosum. Carbohydr. Res. 336, 155-159.

Matsubara, K., Xue, C., Zhao, X., Mori, M., Sugawara, T. and Hirata, T. (2005). Effects of middle molecular weight fucoidans on in vitro and ex vivo angiogenesis of endothelial cells. Int. J. Mol. Med. 15, 695-699.

Nardella, A., Chaubet, F., Sinquin, C., Colliec Jouault, S., Boisson-Vidal, C., Durand, P. and Jozefonvicz, J. (2000). Method for Obtaining Sulfated Polysaccharides. United States Patent 6,028,191.

Patankar, M. S., Oehninger, S., Barnett, T., Williams, R. L. and Clark, G. F. (1993). A revised structure for fucoidan may explain some of its biological activities. J. Biol. Chem. 268, 21770-21776.

Pineda, M. H. (1989). Female reproductive system. In
Veterinary Endocrinology and Reproduction (L. E. McDonald and M. H. Pineda, Eds.). pp. 303-354. Lea \& Febiger, Philadelphia.

Senni, K., Gueniche, F., Foucault-Bertaud, A., Igondjo-Tchen, S., Fioretti, F., Colliec-Jouault, S., Durand, P., Guezennec, J., Godeau, G. and Letourneur, D. (2006). Fucoidan a sulfated polysaccharide from brown algae is a potent modulator of connective tissue proteolysis. Arch. Biochem. Biophys. 445, 56-64.

Shimizu, J., Wada-Funada, U., Mano, H., Matahira, Y., Kawaguchi, M. and Wada, M. (2005). Proportion of murine cytotoxic $\mathrm{T}$ cells is increased by high molecular-weight fucoidan extracted from Okinawa mozuku (Cladosiphon okamuranus). J. Health Sci. 51, 394-397.

Tajima, Y. (1989). Biological reference data book on experimental animals. Soft Science Inc, Tokyo.

Zemani, F., Benisvy, D., Galy-Fauroux, I., Lokajczyk, A., ColliecJouault, S., Uzan, G., Fischer, A. M. and Boisson-Vidal, C. (2005). Low-molecular-weight fucoidan enhances the proangiogenic phenotype of endothelial progenitor cells. Biochem. Pharmacol. 70, 1167-1175. 